\title{
A Derivation and Validation Study of an Early Blood Transfusion Needs Score for Severe Trauma Patients
}

\author{
Hao Wanga, b, Johnbosco Umejiego ${ }^{\text {a }}$, Richard D. Robinson ${ }^{\mathrm{a}}$, Chet D. Schrader ${ }^{\mathrm{a}}$, JoAnna Leuck ${ }^{\mathrm{a}}$, \\ Michael Barra $^{a}$, Stefan Buca ${ }^{a}$, Andrew Shedd ${ }^{a}$, Andrew Bui ${ }^{\text {a }}$, Nestor R. Zenarosa ${ }^{\text {a }}$
}

\begin{abstract}
Background: There is no existing adequate blood transfusion needs determination tool that Emergency Medical Services (EMS) personnel can use for prehospital blood transfusion initiation. In this study, a simple and pragmatic prehospital blood transfusion needs scoring system was derived and validated.
\end{abstract}

Methods: Local trauma registry data were reviewed retrospectively from 2004 through 2013. Patients were randomly assigned to derivation and validation cohorts. Multivariate logistic regression was used to identify the independent approachable risks associated with early blood transfusion needs in the derivation cohort in which a scoring system was derived. Sensitivity, specificity, and area under the receiver operational characteristic (AUC) were calculated and compared using both the derivation and validation data.

Results: A total of 24,303 patients were included with 12,151 patients in the derivation and 12,152 patients in the validation cohorts. Age, penetrating injury, heart rate, systolic blood pressure, and Glasgow coma scale (GCS) were risks predictive of early blood transfusion needs. An early blood transfusion needs score was derived. A score $>$ 5 indicated risk of early blood transfusion need with a sensitivity of $83 \%$ and a specificity of $80 \%$. A sensitivity of $82 \%$ and a specificity of $80 \%$ were also found in the validation study and their AUC showed no statistically significant difference (AUC of the derivation $=0.87$ versus $\mathrm{AUC}$ of the validation $=0.86, \mathrm{P}>0.05)$.

Conclusions: An early blood transfusion scoring system was derived and internally validated to predict severe trauma patients requiring blood transfusion during prehospital or initial emergency department resuscitation.

Keywords: Blood transfusion; Needs; Score; Derivation; Validation; Trauma; Prehospital; Emergency department

Manuscript accepted for publication May 26, 2016

aDepartment of Emergency Medicine, Integrative Emergency Services, John Peter Smith Health Network, 1500 S. Main St., Fort Worth, TX 76104, USA ${ }^{b}$ Corresponding Author: Hao Wang, Department of Emergency Medicine, Integrative Emergency Services, John Peter Smith Health Network, 1500 S. Main St., Fort Worth, TX 76104, USA. Email: hwang01@jpshealth.org

doi: http://dx.doi.org/10.14740/jocmr2598w

\section{Introduction}

Trauma is one of the most common causes of hospital admission and in-hospital mortality [1-3]. Recently, the mortality rate in severe trauma patients has decreased [4-6] due to emphasis on damage control resuscitation (DCR). Blood product transfusion plays an important role in early DCR and improved survival $[7,8]$. In a randomized trial, Del Junco et al (2013) reported that early blood product transfusions during prehospital transportation improved patient outcomes including improved patient acid-base status, decreased blood product usage, and reduced early in-hospital mortality [9]. Other observational studies showed similar benefits among patients who received early blood transfusions either during prehospital or initial emergency department (ED) resuscitation phases [10-12]. These studies documented improved oxygen carrying capacity, more efficient intravascular volume expansion, and reduced risk of early trauma induced coagulopathy [10-12] among patients receiving early blood transfusions. Thus, early prehospital blood product transfusion is an appropriate intervention and improves patient outcomes in the setting of severe trauma patients with high risk of massive hemorrhage. However, criteria available to guide decisions regarding early blood product transfusions among trauma patients vary in published reports $[12,13]$. Variables that were used in some studies (such as imaging, blood gas, lactate, etc.) may not be feasible on scene or reliably adapted by EMS personnel without specific training including the use of focused assessment with sonography for trauma (FAST) $[13,14]$.

In recent years, several blood transfusion scoring systems were derived to predict the need for massive blood transfusion in trauma patients. These tools perform at different levels of sensitivity and specificity [15-19]. Some variables such as blood pressure, heart rate, mechanisms of injury, severity of injuries, and shock index were commonly used for blood transfusion prediction $[18,20]$. Other factors such as FAST results, the occurrence of pelvic trauma, lactate level, and mental status were also used as valuable predictors [14, 17]. The majority of these blood transfusion scores were derived as prerequisites for massive blood transfusion protocols which may not apply universally to patients requiring blood transfusions in general. Some scores require more detailed information (e.g., FAST results, presence of pelvic trauma, etc.) that are not available or noted at the scene $[14,15]$. Several previous studies attempted 
to develop a blood transfusion score to determine appropriate prehospital blood transfusion needs in severe trauma patients [21], but there is no validated study to use as a reference. To fill this gap, this study derived and validated a simple and pragmatic prehospital blood transfusion scoring system designed to determine blood transfusion needs in trauma patients. EMS personnel can easily use this quick scoring system at the scene with limited information on hand.

\section{Methods}

\section{Participant selection}

A retrospective review of local trauma registry for the period January 2004 through December 2013 was performed. Analysis included data associated with trauma patients transported to the study center ED (level 1 trauma center). This study focused on the indication of time-sensitive blood product transfusions either at the scene, en route to ED, or during early ED (defined as the initial $4 \mathrm{~h}$ of ED stay) resuscitations.

\section{Inclusion criteria}

Patients who received any blood products before final ED dispositions were included in the experimental group while those who did not receive any blood products before final ED dispositions were included in the control group. To minimize patient selection bias, all trauma patients were included in this study regardless of mode of transportation (e.g., ground, helicopter, private vehicles, ambulatory, etc.) because some patients received blood products emergently (within $4 \mathrm{~h}$ upon arrival) in the ED although they were not transported by EMS.

\section{Exclusion criteria}

Since this study enrolled all intend-to-treat trauma patients in the local registry, no patients were excluded from the study.

\section{Study design and protocol}

Since this study intended to identify trauma patients who may require earlier blood transfusion, patients who received any blood products during prehospital transportation or within the first $4 \mathrm{~h}$ of ED arrival were screened as positive for early transfusion. Therefore, two groups were studied (early blood transfusion versus non-early blood transfusion groups). Those who did not receive any blood products, or received blood products later in their hospital stay (after $4 \mathrm{~h}$ of arrival) were included in the non-early transfusion group. Early transfusions were defined as those occurring within $4 \mathrm{~h}$ of arrival because the majority of our severe trauma patients remained less than $4 \mathrm{~h}$ at ED and the primary end point of this study was final ED disposition. Blood products included fresh frozen plasma, platelets, whole blood, or packed red blood cells (PRBC). Variables not applicable during the prehospital phase were excluded from this study (e.g. FAST, pelvic trauma sustained). Basic patient demographics (age, sex, and race/ethnicity) and clinical variables (initial EMS/ED vital signs, EMS/ED Glasgow coma scale (GCS), and mechanisms of injury) were analyzed and compared between these two groups. Given that a number of patients receiving blood transfusions were not transferred by EMS, excluding these patients would cause selection bias. The EMS and ED vital signs were compared and an analysis determined whether initial ED vital signs could be used instead of EMS vital signs. This allowed for expansion of our patient population to all severe trauma patients regardless of mode of transportation. To determine the independent risks predictive of early blood product transfusions in the study population, a multivariate logistic regression model was used. Finally, a scoring system was derived and validated internally for identifying severe trauma patients in the field who qualified for early blood product transfusions. The local institutional review board (IRB) approved this study.

\section{Deriving and validating a scoring system for early blood transfusion}

To identify trauma patients who qualified for early blood transfusion, retrospective derivation and validation of a blood transfusion scoring system was implemented. Trauma registry data were randomly split into equal sizes for derivation and validation. Variables chosen for model building were reviewed by clinicians experienced in acute trauma care to ensure consistent clinical significance while protecting against over-fitting. To avoid redundant variables in the final model, Spearman correlation and a regression with variance inflation (VIF) option including all predictive variables was used. The model's discrimination was summarized using the area under the receiver operational characteristic curve (AUC) and the model goodness of fit was measured using the Hosmer and Lemeshow test. Accuracy of the prediction was reported with sensitivity, specificity, positive and negative likelihood ratios. Multiple imputation [22] was applied to handle missing data in the registry. A simple point scoring system was derived using the methods described by Framingham [23]. Scores were calculated among all validated data as well using the same method. Finally, the sensitivity, specificity, and AUC were compared between derivation versus validation data. Repeated analyses were done using different randomized data to determine the stability of the scoring system.

\section{Other statistical analyses}

Student's $t$-test was used to compare continuous variables, while analysis of variance with Bonferroni correction was used to analyze differences among groups. Pearson Chi-square $\left(\chi^{2}\right)$ analysis was used to compare categorical variables. The derivation and validation data were compared to determine patient selection variability. Cronbach's alpha $(\alpha)$ coefficient was measured to determine reliability and consistency of in- 
Table 1. General Information of the Study Population

\begin{tabular}{|c|c|c|c|}
\hline & $\begin{array}{l}\text { Patients receiving blood } \\
\text { transfusions }(\mathrm{N}=784)\end{array}$ & $\begin{array}{l}\text { Patients not receiving } \\
\text { blood transfusions }(\mathrm{N}=\mathbf{2 3 , 4 9 4 )}\end{array}$ & P value \\
\hline Age (mean, SD), years & $40(18)$ & $40(18)$ & 0.44 \\
\hline Gender (male), n (\%) & $604(77)$ & $17,123(73)$ & 0.01 \\
\hline Race/ethnicity, n (\%) & & & 0.09 \\
\hline Black & $119(15)$ & $3,702(16)$ & \\
\hline White & $455(58)$ & $12,830(55)$ & \\
\hline Hispanic & $159(20)$ & $5,747(24)$ & \\
\hline Mode of arrival, $\mathrm{n}(\%)$ & & & $<0.01$ \\
\hline Ambulance/helicopter & 739 (94) & $17,953(76)$ & \\
\hline Private vehicle & $42(5.4)$ & $5,183(22)$ & \\
\hline Type of injury, n (\%) & & & $<0.01$ \\
\hline Blunt & $530(68)$ & $20,005(85)$ & \\
\hline Penetrating & $254(32)$ & $3,289(14)$ & \\
\hline ED vital signs, mean (SD) & & & $<0.01$ \\
\hline Systolic blood pressure $(\mathrm{mm} \mathrm{Hg})$ & $85(45)$ & $133(29)$ & \\
\hline Heart rate $(\mathrm{bpm})$ & $94(45)$ & $89(23)$ & \\
\hline Glasgow coma scale, mean (SD) & $9(5)$ & $14(3)$ & $<0.01$ \\
\hline ED revised trauma scale & & & $<0.01$ \\
\hline Mean (SD) & $4.94(2.71)$ & $7.43(1.28)$ & \\
\hline Median (IQR) & $5.66(2.93-7.84)$ & $7.84(7.84-7.84)$ & \\
\hline ED injury severity scale & & & $<0.01$ \\
\hline Mean (SD) & $27(18)$ & $11(10)$ & \\
\hline Median (IQR) & $25(14-35)$ & $9(4-16)$ & \\
\hline
\end{tabular}

$\mathrm{N}$ : number; ED: emergency department; SD: standard deviation; IQR: interquartile range.

terchanging ED vital signs with EMS vital signs. In general, $\alpha \geq 0.7$ was considered reliable. Independent clinical variables were initially entered into a univariate logistic regression analysis followed by multivariate logistic regression analysis. All descriptive and statistical analyses were conducted using SAS/ STAT 9.2 (Cary, NC) and STATA 12.0 (College Station, TX). A $\mathrm{P}$ value $<0.05$ was considered statistically significant.

\section{Results}

A total of 24,303 patients were listed in the trauma registry during the study period. A subgroup of 784 patients received blood product transfusions either during the prehospital period or within $4 \mathrm{~h}$ of arrival to ED resulting in a transfusion rate of $3.23 \%$. The missing data rate ranged $0-9.4 \%$ among main variables and 1.8-11.7\% among other variables (Supplementary Table 1, www.jocmr.org). Since this study focused on the prediction of blood transfusion among trauma patients, 25 patients with unknown information on blood transfusion were excluded from the final analysis. Table 1 describes the general information among patients who received blood product transfusions versus those not receiving transfusions. Patients who received blood transfusion were predominately male, arrived via ambulance/helicopter, sustained more penetrating injuries, had more severe injuries, and were noted to have less stable vital signs. As compared to patients who did not receive blood products, the majority of these variables demonstrated significant differences except for age and race/ethnicity (Table 1).

In order to determine whether prehospital vital signs (e.g. pulse, systolic blood pressure (SBP), and GCS) could be replaced by ED vital signs, Cronbach's alpha $(\alpha)$ coefficient was measured to determine the reliability and consistency of these exchanges. Reliability coefficients $(\alpha)$ measured were GCS = 0.99 , pulse $=0.70$, and $\mathrm{SBP}=0.69$, indicating strong reliability between prehospital and ED vital signs.

To derive and validate a blood transfusion needs scoring system, registry data were randomly split into two equal parts (Supplementary Table 2, www.jocmr.org). Our findings demonstrated no significant differences between derivation and validation data, indicating no significant patient selection bias between the two datasets. When derivation data were used for identifying independent risk factors, patient age, GCS, penetrating injury, initial ED pulse, and SBP were considered statistically significant. Odds ratios of each risk factor (Table 2) were determined using either univariate or multivariate logistic regression analysis with the final model of C-statistic (AUC) equal to 0.90 (95\% confidence interval: $0.89-0.92)$. Results 
Table 2. Independent Risk Factors Predictive of Early Blood Transfusion Needs Using Derivation Dataset

\begin{tabular}{llll}
\hline & Unadjusted odds ratios $\mathbf{( 9 5 \%} \mathbf{C I})$ & Adjusted odds ratios $\mathbf{( 9 5 \%}$ CI) & C-statistics \\
\hline Age & $1.14(0.98-1.34)$ & $1.02(1.01-1.03)$ & 0.53 \\
Penetrating injury & $2.90(2.34-3.60)$ & $2.07(1.59-2.71)$ & 0.59 \\
ED pulse & $1.01(1.00-1.01)$ & $1.03(1.03-1.04)$ & 0.60 \\
ED GCS & $0.79(0.78-0.81)$ & $0.85(0.83-0.87)$ & 0.78 \\
ED SBP & $0.97(0.97-0.97)$ & $0.97(0.96-0.97)$ & 0.85 \\
The final model & & & 0.90 \\
\hline
\end{tabular}

CI: confidence interval; ED: emergency department; SBP: systolic blood pressure; GCS: Glasgow coma scale.

of the Hosmer-Lemeshow goodness of fit test for logistic regression confirmed that the distribution fit the data well and the risk prediction was well calibrated $\left(\chi^{2}(8)=11.57, \mathrm{P}=0.172\right)$. These five risk factors (patient age, GCS, penetrating injury, initial ED pulse, and SBP) were then incorporated into the blood transfusion score with an associated integer point value (Table 3 ) resulting in a total score ranging from -4 to 17 . In order to confirm the accuracy of using the study transfusion score, this score was applied back to the derivation data yielding a similar AUC (0.87, 95\% confidence interval: 0.85 - 0.89) when compared to the AUC determined by the logistic regression model.

A validation dataset was used for internal validation yielding an AUC of 0.86 (95\% confidence interval: 0.84 - 0.88). No statistically significant difference was found between the derivation and validation data (Fig. $1, \mathrm{P}>0.05$ ). Using a score $>5$ as the predictive "cutoff value" for early blood transfusion needs, the sensitivity and specificity of the derivation data

Table 3. Early Blood Transfusion Needs Score

\begin{tabular}{|c|c|}
\hline Risk factors & Score \\
\hline \multicolumn{2}{|l|}{ Age (years) } \\
\hline $0-55$ & 0 \\
\hline $56-70$ & 1 \\
\hline$>70$ & 2 \\
\hline \multicolumn{2}{|l|}{ Type of injury } \\
\hline Penetrating injury & 2 \\
\hline Non-penetrating injury & 0 \\
\hline \multicolumn{2}{|l|}{ Pulse (beats/min) } \\
\hline$<60$ & -4 \\
\hline $60-119$ & 0 \\
\hline$\geq 120$ & 3 \\
\hline \multicolumn{2}{|c|}{ Systolic blood pressure (mm Hg) } \\
\hline$<90$ & 7 \\
\hline$\geq 90$ & 0 \\
\hline \multicolumn{2}{|l|}{ Glasgow coma scale } \\
\hline $3-8$ & 3 \\
\hline $9-13$ & 1 \\
\hline $14-15$ & 0 \\
\hline Total score & $-4-17$ \\
\hline
\end{tabular}

were $83 \%$ and $80 \%$, respectively. Similar results were obtained when analyzing the validation data (Supplementary Table 3, www.jocmr.org). Moreover, if a score $>8$ was considered as the predictive "cutoff value" for early blood transfusion needs, derivation data set analysis specificity was about $90 \%$, whereas sensitivity dropped to near $70 \%$. Validation data set analysis yielded similar results (Supplementary Table 3, www.jocmr. org). Our studies suggest in general that a score of $>5$ be considered for patients with potential need for early blood transfusion and a score of $>8$ be considered for high risk patients. Repeat analyses were performed using different randomized data in the derivation and validation sets yielding similar results indicating adequate stability of the study scoring system (data not shown).

\section{Discussion}

We derived and internally validated an early blood transfusion needs scoring system. Trauma patients with higher scores had higher correlation of receiving blood transfusions earlier in their care timelines. Our study recommends strong consideration of EMS initiated prehospital blood transfusion for patients with high blood transfusion needs scores. Variables used in this scoring system are easy to harvest thereby facilitating a pragmatic protocol which may improve patient outcomes. The significance of this study is that the early blood transfusion needs score will provide appropriate guidance to EMS personnel implementing DCR in the field. Evidence from previous studies supported blood product transfusions being one of the most effective interventions during initial DCR and proving to be particularly beneficial in severe trauma patients $[10,13$, 24]. This strategy can shorten length of stay in hospital, protect against acute traumatic coagulopathy, and reduce in-hospital and 30-day mortality [12, 13]. Early blood transfusions may subsequently decrease the total amount of blood transfusion requirements per patient [13]. When considering poor outcomes observed among trauma patients with delayed blood transfusions during the initial resuscitation and among trauma patients receiving delayed excessive and unnecessary or futile blood transfusions $[10,11,25,26]$, it is apparent that a strategy that balances the timely delivery of blood products produces improved patient outcomes [27]. Given limited on scene information, the intricate challenge for EMS personnel and physicians is to determine which specific patients require early blood 


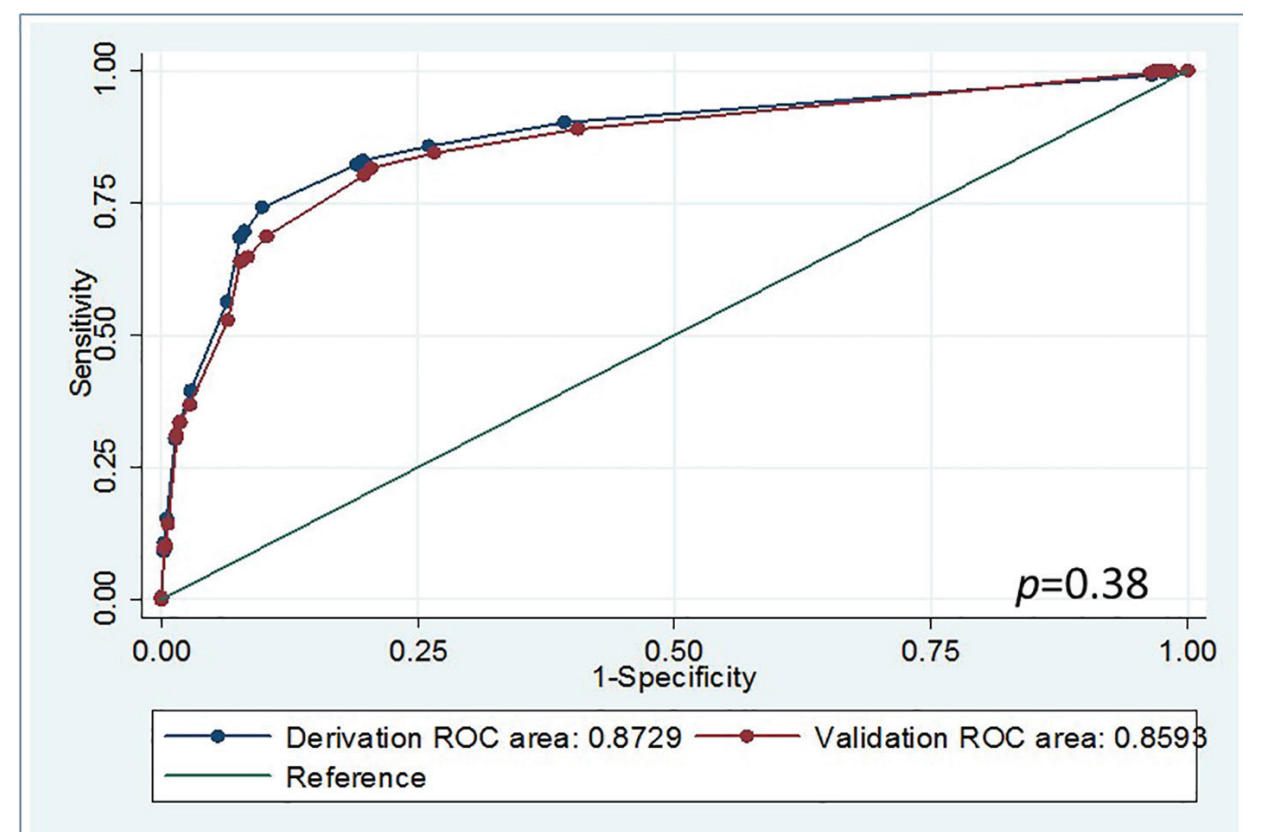

Figure 1. Receiver operating characteristic curve comparisons between deviation and validation data using blood transfusion scoring system.

transfusions in the prehospital setting. In general, time sensitive decisions are best supported by simple, easy-to-use tools as compared to those requiring sophisticated calculations. In this study, we show unequivocally that all variables required for blood transfusion needs score calculations can be obtained within a few minutes in the field. These data are readily and accurately obtained by EMS personnel having received minimal training. The variables used in the calculation of blood transfusion needs scores in this study have been reported previously in the literature [15, 17-19]. Among these variables, SBP and heart rate (HR) are two variables commonly used to determine the need for blood transfusion in the literature $[12,28]$. Isolated hypotension was found to be less reliable unless combined with additional variables (such as age, penetrating injury, HR, etc.) [29]. Some studies reported HR can be masked by medication effects especially among geriatric patients with chronic medical conditions $[30,31]$. Of interest a recent report on geriatric trauma patients with pre-injury cardiac medications (e.g. beta-blocker) did not seem to blunt hyper-dynamic responses [32]. In some studies, shock index (e.g. HR/SBP) was determined to be more accurate and a better predictor of early blood transfusion needs [33-35]. However, a recent study [36] showed shock index had less clinical predictive power. Results from an analysis of the national trauma data bank also showed a combination of SI and age is a better predictor of life threatening shock in older trauma patients [37]. Generally speaking, GCS is less reliable in determining the need for blood transfusion on its own, but has clinical value when considered along with other variables [38]. Taken together, it is ideal to derive a tool with a combination of all available information while also addressing the individual weight of each variable to increase the probability of predicting time-sensitive interventions.

In this study, our blood transfusion needs score demon- strated approximately $80 \%$ sensitivity and specificity. However, concern regarding outcomes of patients receiving blood transfusions based on pseudo negative/positive score results cannot be ruled out. A few studies reported unnecessary blood transfusions with no significant harmful outcomes $[39,40]$. If indicated, emergency physicians may decide to stop the prehospital initiated blood transfusion upon patient arrival or when further detailed information is obtained (e.g., imaging studies). Future research is needed to further delineate the risks of initiating limited unnecessary blood transfusions.

\section{Limitation}

This is a retrospective study with limited information, missing data and potential for patient population selection bias. However, the overall missing data rate among the major variables was less than 10\% (Supplementary Table 1, www.jocmr.org). Data imputed in this study posed less significant bias with respect to incomplete data (Supplementary Table 1, www.jocmr. org). We were not able to include all possible variables that may predict blood transfusions needs. The simplest variables that were easily approachable were used, therefore, vital signs, age, and mental status were chosen. ED vital signs as opposed to vital signs at the scene were used for blood transfusion needs prediction which may be a source of bias. This was done to include patients who were not transported by EMS but still required early blood transfusion during ED resuscitation. Our EMS vital signs correlated well with the ED vital signs within the same categorical range due to limited EMS transportation time in this study (usually less than $10-15 \mathrm{~min}$ ). The use of initial ED vital signs is acceptable although future studies with external validation of a larger sample size are recommended. 


\section{Conclusion}

An early blood transfusion needs scoring system was derived and internally validated to predict severe trauma patients requiring blood transfusion during prehospital or initial ED resuscitation. A score $>5$ indicated risk of receiving early blood transfusion with about $80 \%$ sensitivity and specificity in both the derivation and validation cohorts.

\section{Acknowledgments}

The authors would like to thank Shrivastav M and Phillips J for editorial assistance.

\section{Conflicts of Interest}

None.

\section{Author Contributions}

HW, RDR, and CDS conceived the study and developed the design in consultation with all of the authors. JU, MB, and $\mathrm{AB}$ assembled the dataset and collected the data. HW, JU, and RDR conducted the statistical analyses. HW, RDR, JL, SB, AS, and NRZ drafted the articles. All authors read and approved the final manuscript. HW takes responsibility for the paper as a whole.

\section{References}

1. Wang H, Phillips JL, Robinson RD, Duane TM, Buca S, Campbell-Furtick MB, Jennings A, et al. Predictors of mortality among initially stable adult pelvic trauma patients in the US: Data analysis from the National Trauma Data Bank. Injury. 2015;46(11):2113-2117.

2. Minei JP, Fabian TC, Guffey DM, Newgard CD, Bulger EM, Brasel KJ, Sperry JL, et al. Increased trauma center volume is associated with improved survival after severe injury: results of a Resuscitation Outcomes Consortium study. Ann Surg. 2014;260(3):456-464; discussion 464455.

3. Schoeneberg C, Probst T, Schilling M, Wegner A, Hussmann B, Lendemans S. Mortality in severely injured elderly patients: a retrospective analysis of a German level 1 trauma center (2002-2011). Scand J Trauma Resusc Emerg Med. 2014;22:45.

4. Langan NR, Eckert M, Martin MJ. Changing patterns of in-hospital deaths following implementation of damage control resuscitation practices in US forward military treatment facilities. JAMA Surg. 2014;149(9):904-912.

5. Brinck T, Handolin L, Lefering R. The Effect of Evolving Fluid Resuscitation on the Outcome of Severely Injured Patients: An 8-year Experience at a Tertiary Trauma Center. Scand J Surg. 2016;105(2):109-116.
6. Shrestha B, Holcomb JB, Camp EA, Del Junco DJ, Cotton BA, Albarado R, Gill BS, et al. Damage-control resuscitation increases successful nonoperative management rates and survival after severe blunt liver injury. $\mathrm{J}$ Trauma Acute Care Surg. 2015;78(2):336-341.

7. Riskin DJ, Tsai TC, Riskin L, Hernandez-Boussard T, Purtill M, Maggio PM, Spain DA, et al. Massive transfusion protocols: the role of aggressive resuscitation versus product ratio in mortality reduction. J Am Coll Surg. 2009;209(2):198-205.

8. Dente CJ, Shaz BH, Nicholas JM, Harris RS, Wyrzykowski AD, Patel S, Shah A, et al. Improvements in early mortality and coagulopathy are sustained better in patients with blunt trauma after institution of a massive transfusion protocol in a civilian level I trauma center. $\mathbf{J}$ Trauma. 2009;66(6):1616-1624.

9. del Junco DJ, Holcomb JB, Fox EE, Brasel KJ, Phelan HA, Bulger EM, Schreiber MA, et al. Resuscitate early with plasma and platelets or balance blood products gradually: findings from the PROMMTT study. J Trauma Acute Care Surg. 2013;75(1 Suppl 1):S24-30.

10. Brown JB, Cohen MJ, Minei JP, Maier RV, West MA, Billiar TR, Peitzman AB, et al. Pretrauma center red blood cell transfusion is associated with reduced mortality and coagulopathy in severely injured patients with blunt trauma. Ann Surg. 2015;261(5):997-1005.

11. Brown JB, Sperry JL, Fombona A, Billiar TR, Peitzman AB, Guyette FX. Pre-trauma center red blood cell transfusion is associated with improved early outcomes in air medical trauma patients. J Am Coll Surg. 2015;220(5):797-808.

12. O'Reilly DJ, Morrison JJ, Jansen JO, Apodaca AN, Rasmussen TE, Midwinter MJ. Prehospital blood transfusion in the en route management of severe combat trauma: a matched cohort study. J Trauma Acute Care Surg. 2014;77(3 Suppl 2):S114-120.

13. Holcomb JB, Donathan DP, Cotton BA, Del Junco DJ, Brown G, Wenckstern TV, Podbielski JM, et al. Prehospital Transfusion of Plasma and Red Blood Cells in Trauma Patients. Prehosp Emerg Care. 2015;19(1):1-9.

14. Hsu JM, Hitos K, Fletcher JP. Identifying the bleeding trauma patient: predictive factors for massive transfusion in an Australasian trauma population. J Trauma Acute Care Surg. 2013;75(3):359-364.

15. Rainer TH, Ho AM, Yeung JH, Cheung NK, Wong RS, Tang N, Ng SK, et al. Early risk stratification of patients with major trauma requiring massive blood transfusion. Resuscitation. 2011;82(6):724-729.

16. Yumoto T, Iida A, Hirayama T, Tsukahara K, Shiba N, Yamanouchi H, Sato K, et al. Immediate screening method for predicting the necessity of massive transfusions in trauma patients: a retrospective single-center study. J Intensive Care. 2014;2(1):54.

17. Ogura T, Nakamura Y, Nakano M, Izawa Y, Nakamura M, Fujizuka K, Suzukawa M, et al. Predicting the need for massive transfusion in trauma patients: the Traumatic Bleeding Severity Score. J Trauma Acute Care Surg. 2014;76(5):1243-1250.

18. Cotton BA, Dossett LA, Haut ER, Shafi S, Nunez TC, 
Au BK, Zaydfudim V, et al. Multicenter validation of a simplified score to predict massive transfusion in trauma. J Trauma. 2010;69(Suppl 1):S33-39.

19. Rahbar MH, del Junco DJ, Huang H, Ning J, Fox EE, Zhang X, Schreiber MA, et al. A latent class model for defining severe hemorrhage: experience from the PROMMTT study. J Trauma Acute Care Surg. 2013;75(1 Suppl 1):S82-88.

20. Nunez TC, Voskresensky IV, Dossett LA, Shinall R, Dutton WD, Cotton BA. Early prediction of massive transfusion in trauma: simple as ABC (assessment of blood consumption)? J Trauma. 2009;66(2):346-352.

21. Tonglet ML, Minon JM, Seidel L, Poplavsky JL, Vergnion M. Prehospital identification of trauma patients with early acute coagulopathy and massive bleeding: results of a prospective non-interventional clinical trial evaluating the Trauma Induced Coagulopathy Clinical Score (TICCS). Crit Care. 2014;18(6):648.

22. Rubin DB, Schenker N. Multiple imputation in healthcare databases: an overview and some applications. Stat Med. 1991;10(4):585-598.

23. Sullivan LM, Massaro JM, D'Agostino RB, Sr. Presentation of multivariate data for clinical use: The Framingham Study risk score functions. Stat Med. 2004;23(10):16311660 .

24. Bodnar D, Rashford S, Williams S, Enraght-Moony E, Parker L, Clarke B. The feasibility of civilian prehospital trauma teams carrying and administering packed red blood cells. Emerg Med J. 2014;31(2):93-95.

25. Holena DN, Netzer G, Localio R, Gallop RJ, Bellamy SL, Meyer NJ, Shashaty MG, et al. The association of early transfusion with acute lung injury in patients with severe injury. J Trauma Acute Care Surg. 2012;73(4):825-831.

26. Perel P, Clayton T, Altman DG, Croft P, Douglas I, Hemingway $\mathrm{H}$, Hingorani A, et al. Red blood cell transfusion and mortality in trauma patients: risk-stratified analysis of an observational study. PLoS Med. 2014;11(6):e1001664.

27. Boutefnouchet T, Gregg R, Tidman J, Isaac J, Doughty H. Emergency red cells first: Rapid response or speed bump? The evolution of a massive transfusion protocol for trauma in a single UK centre. Injury. 2015;46(9):17721778.

28. Brown JB, Guyette FX, Neal MD, Claridge JA, Daley BJ, Harbrecht BG, Miller RS, et al. Taking the Blood Bank to the Field: The Design and Rationale of the Prehospital Air Medical Plasma (PAMPer) Trial. Prehosp Emerg Care. 2015;19(3):343-350.

29. Newgard CD, Meier EN, McKnight B, Drennan IR, Richardson D, Brasel K, Schreiber M, et al. Understanding traumatic shock: out-of-hospital hypotension with and without other physiologic compromise. J Trauma Acute Care Surg. 2015;78(2):342-351.

30. Neideen T, Lam M, Brasel KJ. Preinjury beta blockers are associated with increased mortality in geriatric trauma patients. J Trauma. 2008;65(5):1016-1020.

31. Heffernan DS, Thakkar RK, Monaghan SF, Ravindran R, Adams CA, Jr., Kozloff MS, Gregg SC, et al. Normal presenting vital signs are unreliable in geriatric blunt trauma victims. J Trauma. 2010;69(4):813-820.

32. Evans DC, Khoo KM, Radulescu A, Cook CH, Gerlach AT, Papadimos TJ, Steinberg SM, et al. Pre-injury beta blocker use does not affect the hyperdynamic response in older trauma patients. J Emerg Trauma Shock. 2014;7(4):305-309.

33. Olaussen A, Blackburn T, Mitra B, Fitzgerald M. Review article: shock index for prediction of critical bleeding post-trauma: a systematic review. Emerg Med Australas. 2014;26(3):223-228.

34. Olaussen A, Peterson EL, Mitra B, O'Reilly G, Jennings PA, Fitzgerald M. Massive transfusion prediction with inclusion of the pre-hospital Shock Index. Injury. 2015;46(5):822-826.

35. Vandromme MJ, Griffin RL, Kerby JD, McGwin G, Jr., Rue LW, 3rd, Weinberg JA. Identifying risk for massive transfusion in the relatively normotensive patient: utility of the prehospital shock index. J Trauma. 2011;70(2):384388; discussion 388-390.

36. Guyette FX, Meier EN, Newgard C, McKnight B, Daya M, Bulger EM, Powell JL, et al. A comparison of prehospital lactate and systolic blood pressure for predicting the need for resuscitative care in trauma transported by ground. J Trauma Acute Care Surg. 2015;78(3):600-606.

37. Zarzaur BL, Croce MA, Magnotti LJ, Fabian TC. Identifying life-threatening shock in the older injured patient: an analysis of the National Trauma Data Bank. J Trauma. 2010;68(5):1134-1138.

38. Elterman J, Brasel K, Brown S, Bulger E, Christenson J, Kerby JD, Kannas D, et al. Transfusion of red blood cells in patients with a prehospital Glasgow Coma Scale score of 8 or less and no evidence of shock is associated with worse outcomes. J Trauma Acute Care Surg. 2013;75(1):8-14; discussion 14.

39. Mitra B, Nash JL, Cameron PA, Fitzgerald MC, Moloney J, Velmahos GC. Potentially avoidable blood transfusion during trauma resuscitation. Injury. 2015;46(1):10-14.

40. Carson JL, Sieber F, Cook DR, Hoover DR, Noveck H, Chaitman BR, Fleisher L, et al. Liberal versus restrictive blood transfusion strategy: 3-year survival and cause of death results from the FOCUS randomised controlled trial. Lancet. 2015;385(9974):1183-1189. 